\title{
Perceptions and experiences of community health workers on community-based child health interventions in the Maekel region, Eritrea
}

\author{
Jordanos T. McDonald', Jill Trenholm² \\ 1 Department of Global Public Health, Global and Sexual Health Research Group, Karolinska Institutet, Solna, Sweden, ${ }^{2}$ Department of Women's and \\ Children's Health, International Maternal and Child Health, Uppsala University, Uppsala, Sweden \\ Keywords: eritrea, child health, community health workers, integrated community case management
}

https://doi.org/10.29392/001c.24589

\section{Journal of Global Health Reports}

Vol. 5, 2021

\section{Background}

In 2015 alone, 5,9 million children under the age of five died. In an attempt to reduce child mortality in Eritrea, the government implemented community- based child health interventions by community health workers (CHWs). Despite progress, challenges remain. In Eritrea, few studies have examined CHWs and community perceptions of child health interventions. The aim of this study was to explore the perceptions of CHWs regarding benefits and challenges of community-based child health interventions in the Maekel region in Eritrea.

\section{Methods}

An explorative qualitative study was conducted employing focus group discussions. Data was analyzed using qualitative content analysis.

\section{Results}

The CHWs reported positive aspects such as easier access to health services, generated relationships and support and opportunities to give back to the community. Perceived challenges were: constraints in delivering services, resources and supervision, inadequate knowledge and awareness and juggling multiple roles with no pay. The ministry of health $(\mathrm{MoH})$ in Eritrea prioritizes reducing child mortality but, is still in the process of building and stabilizing the healthcare system. The biggest challenge is to incorporate community level Integrated Management of Childhood Illnesses and Case Management (C-IMCI/ iCCM), involving communities, families and CHWs into the current system of limited resources.

\section{Conclusions}

This study indicates the importance of focusing on identifying solutions to the challenges the CHWs faces and understanding factors associated with CHWs motivation. This with the hope of better effectiveness and sustainability of the C-IMCI/iCCM program.

Globally, 5,9 million children under the age of five died in 2015 from pneumonia, diarrhea and malaria as the leading causes of mortality. ${ }^{1,2}$ According to the Eritrean population and health survey in 2010, 1 in 16 Eritrean children die before the age of five. The leading causes are neonatal causes, pneumonia, diarrhea and malnutrition, whereas malaria is no longer a major public health concern. The country has however achieved remarkable progress in reducing child mortality with a current under-five child mortality rate of 44 per 1000 live births and an average annual reduction of around $3.4 \%$ since $1995 .{ }^{3-7}$ To further decrease child mortality, Eritrea has implemented child health programs to strengthening care at the community level. Child health interventions were introduced in 2005, in the Debud region by the ministry of health (MoH). In 2006, an evaluation of the community based interventions was conducted and showed increased health care coverage and community support. 4,6 Integrated Management of Childhood Illnesses (IMCI) and Integrated Community Case management (iCCM) of childhood illness are programs developed by the World Health Organization and the United Nations' Children's Fund (WHO/UNICEF). The community aspect of the IMCI (CIMCI) addresses family and community health practices through community health workers (CHW) ${ }^{8-10}$ Evaluations of C-IMCI suggest that the intervention has fallen short; given the low coverage, fragmented health systems and weak health promotion. ${ }^{11}$ Factors such as: sufficient equipment, supervision of $\mathrm{CHW}$ and duration of their training seem to affect their performance and thus the utilization and coverage of the intervention. ${ }^{11}$ The iCCM is based on 
delivering treatment for pneumonia, malaria and diarrhea to children under five and Community Case Management (CCM) amplifies the treatment arm of C-IMCI. ${ }^{2}$ However, inequities in access to treatment, poor health care infrastructure and shortage of staff and equipment has challenged overall iCCM implementation elsewhere, ${ }^{12,13}$ and also in Eritrea. ${ }^{6}$ Within the Eritrean program, CHWs are crucial. Their duties include: conducting awareness and prevention sessions on child health ${ }^{5,7}$ and integrated community case management of pneumonia, malaria and diarrhea. ${ }^{5,7}$ The CHWs classify and treat the child with appropriate interventions, make referrals, counsel the caregivers on appropriate home care and conduct follow-ups to assess the child's treatment completion. The intention of CHWs is to able to provide culturally appropriate health services to the community, of which they are residents of and therefore understand the context. ${ }^{14}$ The $\mathrm{MoH}$, through health centers (HC), is responsible for training CHWs, providing technical assistance, continuous supportive supervision, monitoring and the provision of medical supplies and drugs for their use. $^{5-7}$

Studies carried out regarding child health interventions in Eritrea has mainly been on assessment of the use of clinical IMCI guidelines in children with diarrhea and malaria. ${ }^{4}$ Very few studies have been conducted on community-based child health intervention involving CHWs in Eritrea and there have been no studies on the overall C-IMCI program in the Maekel region. Furthermore, considering that CHWs are the main actors in the program, to our knowledge there have been no studies seeking to understand the CHWs' perceptions and experiences, particularly of working with this program in Eritrea. This study addresses this gap by exploring the perceptions and experiences of CHWs on community-based child health interventions in Eritrea with the goal of furthering the understanding of the benefits, challenges and implementation experiences of the C-IMCI/ iCCM program.

\section{METHODS}

A qualitative research design employing focus group discussions (FGD) was chosen for its ability to illuminate how social norms and perceptions are generated amongst social groups in a particular context. ${ }^{15,16}$ This study was reviewed and approved by the Health research and ethical committee from the $\mathrm{MoH}$ in Asmara (Reference number: 15743/ 4850119).

\section{SETTING}

Eritrea is situated in north eastern Africa ${ }^{3,5}$ and was declared an independent nation in 1993 after being at war with Ethiopia for 30 years. ${ }^{6}$ Since then, efforts have been made to build the health care system and train health care staff, with the aim of achieving equitable distribution.6,7 Between 1995 and 2010 the number of physicians doubled from 3 per 100,000 populations to 6 per 100,000 and the number of nurses increased from 20.5 to 75 per 100,000 populations. Despite the growth of trained healthcare workforce, it is below estimated needs.6,7 Preventive and curative services are provided at the primary level, at health

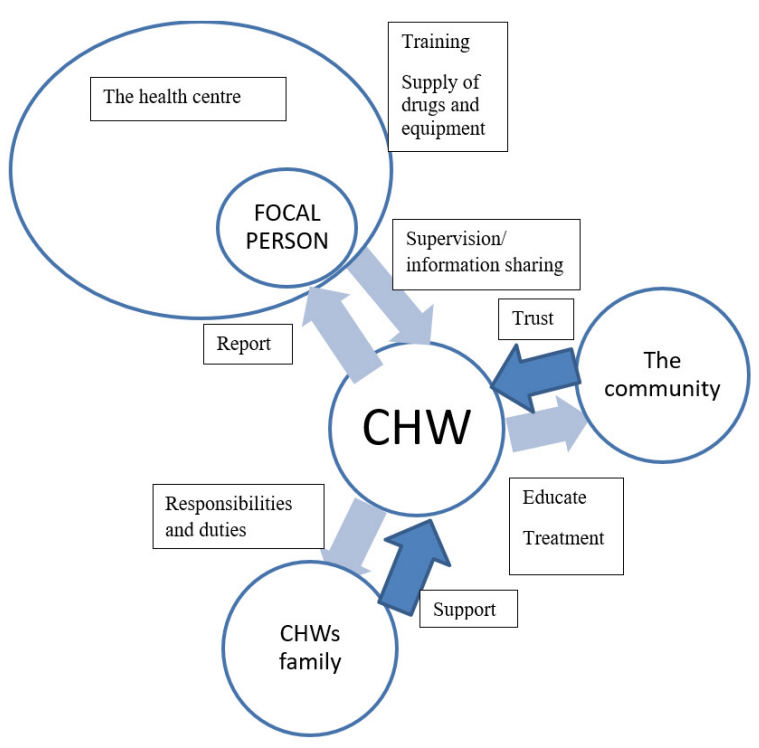

\section{Figure 1. An adopted conceptual map of the $\mathrm{CHW}^{+}$ framework based on the Eritrean context ${ }^{17}$}

CHWs - community health workers.

stations, health centers and community hospitals..$^{5}$ About $15 \%$ of the population is less than 5 years old and $65 \%$ of the population live in rural areas. ${ }^{5}$ This study was conducted in the Maekel region, in the center of Eritrea, where the CIMCI/iCCM program has been implemented since 2013. Before launching the program, the CHWs receive two- weeks of training in preventive and curative interventions involving case management and household health care practices. ${ }^{7}$ The CHWs are volunteers, receiving no payment, living in rural areas, selected by the community, who report monthly prevalence of illness, treatment records and follow ups, to a so-called "focal-person" at each health center (HC) ${ }^{7}$ The focal persons are staff at the $\mathrm{HC}$ responsible of the community-based interventions and the CHWs.

\section{PARTICIPANTS AND SAMPLING}

Participants for the study were recruited using purposive sampling of CHWs in the Maekel region with the assistance of $\mathrm{MoH}$ focal persons. In the Maekel region, there were only one male $\mathrm{CHW}$ at the time. Inclusion criteria consisted of being female or male CHWs who had work experience of more than 18 months in their role. The CHWs were then offered to take part in a FGD. Totally, 19 persons agreed yielding three FGDs with 4-8 participants in each group.

\section{THEORETICAL FRAMEWORK}

Figure 1 shows our adapted framework based on the UNICEF $\mathrm{CHW}^{+}$framework with the addition of factors that emerged through this research. The adapted conceptual map helped organize the data and guided the interpretation during data analysis. ${ }^{17}$ The framework provides an understanding of the different elements of the program, the various inter-relationships and the multi-sectorial approach involved in the CHW led program, from the perspective of the CHWs. ${ }^{17}$ 
Table 1. An examples of a meaning unit, condense meaning unit and code

\begin{tabular}{lll}
\hline Meaning unit & Condense meaning unit & Code \\
\hline $\begin{array}{l}\text { The health centers are far from the } \\
\text { villages and so the CHW are making it } \\
\text { easier for the mothers to get health care } \\
\text { and attention for their children }\end{array}$ & $\begin{array}{l}\text { Far to the health centers and CHWs are } \\
\text { facilitating easier accesses to health } \\
\text { services }\end{array}$ & Easier access to health services \\
\end{tabular}

CHWs - community health workers.

\section{DATA COLLECTION}

Data collection took place in August and September 2015. The primary author and one other researcher, a local lecturer of Epidemiology at University of Asmara, moderated the FGDs with the CHWs in Tigrinya. Since the CHWs lived in rural areas and are difficult to contact, an information sheet about the study was given to the focal-persons at each HC by the principal investigator, which they subsequently shared with the CHWs. Most CHWs walked for hours to participate in a FGDs. After an oral presentation of the study, all participants in agreement were asked to sign a consent form and to fill questions containing demographics (sex, age, education level, occupation) and length of employment. The FGDs followed a semi-structured discussion guide, were audio-taped and lasted 45 minutes to one hour. The main issues investigated were the CHWs perceptions concerning the benefits, challenges and limits of the program. For this study, 3 FGDs were conducted with 19 CHWs. Observation notes were recorded after each FGDs. Additionally, during the two months in the field, the researcher kept a record of reflections and observations in order to follow the audit trail and allow for reflexivity throughout the research process. Participant observation, FGDs transcripts and discussions with the staff at the $\mathrm{MoH}$ and at each $\mathrm{HC}$ promoted data source triangulation.

\section{DATA ANALYSIS}

The FGDs recordings were transcribed verbatim and translated from Tigrinya to English by a trained translator, a lecturer of English at University of Asmara. The principal investigator also listened to all audiotapes to verify the transcripts. Analysis of the data was performed by the first author using Qualitative Content Analysis (QCA). The QCA procedure involves: identifying meaning units or pieces of text that hold meaning, condensation of meaning units into codes and categories. ${ }^{18}$ The coding and eventually findings were further refined thought consensus, after discussions with the research team consisting of seasoned qualitative researchers at Uppsala University. FGD notes supplemented the transcripts providing descriptions of group interactions and body language information. An example of the QCA process is illustrated in Table 1.

\section{PROFILE OF PARTICIPANTS}

Demographic characteristics of the 19 CHWs are summarized in Table 2. All participants but one were women be-
Table 2. Demographic characteristics of CHWs in Maekel Region

\begin{tabular}{lr}
\hline Characteristics & Number of CHWs \\
\hline Sex & 18 \\
Female & 1 \\
Male & \\
Age (years) & \\
$25-30$ & 11 \\
$30-35$ & 3 \\
$35-40$ & 3 \\
Educational level & \\
Elementary & 6 \\
Junior & 4 \\
High school and above & 8 \\
Occupation & \\
House wife & \\
Farmer & 16 \\
Work experience as a CHW & \\
19-20 months & \\
\hline Total & \\
\hline
\end{tabular}

CHWs - community health workers.

tween 25-40 years. Their educational level varied, with 6 completing elementary school, 4 finishing junior high school and 8 completing high school. All participants had received 2 weeks of training in C-IMCI/iCCM in 2013. The majority of the CHWs were housewives with 3 reporting to have farming as their main occupation. Reported length of time working as a CHW ranged from 19-20 months.

\section{RESULTS}

The findings from this study indicated three main benefits of the program: easier access to health services, generated relationships and support and opportunities to give back to the community. There were also challenges, which were as follows: constraints in delivering services, resources and supervision, inadequate knowledge and awareness and juggling multiple roles with no pay (Table 3). Participants are 
identified by their FGD number and a participant letter.

\section{EASIER ACCESS TO HEALTH SERVICES}

Central benefits and positive experiences of the program discussed by the CHWs involved increasing access to free health services to under-served population. They also acknowledged that appropriate treatments were given in the villages and that there are various incentives for the community. These incentives included avoiding long journeys to the health centers, out-of-pocket health expenditures and reduced transportation costs.

'To reduce child mortality, our services are in the villages and free' (FGD1, B)

The CHWs appeared to believe that additional benefits of the program, were services involving counselling the mothers, regular home visits and conducting important followups to check that mothers complied with home-care and recommendations:

I....we go to their homes and check whether the mother is following the instructions or not. We also check if the child is feeling better or not... we then advise the mother' (FGD3, R)

\section{GENERATED RELATIONSHIPS AND SUPPORT}

The CHWs were encouraged by the program benefits they had observed in the community. Generally, they reported positive reception and support by community members, and the support was said to have developed after successful treatments.

'Once a man whose child I treated spread the word all over the village that I had saved his son... after that the mothers started to come' (FGD1, A)

This contributed to more mothers visiting CHWs. The notions of providing curative treatment influenced the communities trust in medical treatment and eventually in the CHWs.

\section{'... mothers come to us believing in the treatment'
$(F G D 1, H)$}

Another positive element described by the CHW is that they greet the mothers in a friendly manner and provide better communication opportunities:

'One of the benefits is the friendly reception that we give the mothers and the children' (FGD2,N)

An additional benefit was the important relation generated between the communities, through CHWs, to the HC. The linkage was said to improve in particular, referrals to the $\mathrm{HC}$ and better communication:

'I refer the patient to th e health center.... They know that we send these mothers, we have an understanding... [Group members nodding]' (FGD3, R)

In regards to supervision, CHWs emphasized receiving good supervision from the focal-persons at the $\mathrm{HC}$ and also expressed good relationships with their supervisor.

\section{Table 3. Perceived benefits and challenges among the} CHWs

\section{Benefits}

- Easier access to health services

- Generated relationships and support

- Opportunities to give back to the community

Challenges

- Constraints in delivering services, resources and supervision

- Inadequate knowledge and awareness

- Juggling multiple roles with no pay

CHWs - community health workers.

'We are given sufficient guidance from the focal person at the health center' (FGD2, L)

\section{OPPORTUNITIES TO GIVE BACK TO THE COMMUNITY}

The CHWs described their work as an action to give back to the community. Thus, they were grateful to have had the opportunity to be trained.

'The training was very effective... you serve the whole vil-
lage ... and this is reason enough for satisfaction' (FGD3,P)

The CHWs reported to understand the health needs of the community. They believed they are the first contact and the only link to the nearest health facility for many in the villages.

\section{| "We are the ones in contact with the people every day"}

\section{CONSTRAINTS IN DELIVERING SERVICES, RESOURCES AND SUPERVISION}

The CHWs described the dilemmas they faced, they elaborated on that key challenges are frequent shortage of drugs and equipment. This has affected their performance and the quality of care they deliver. The CHWs implied long waits for medicines and medical equipment from the health centers.
'....When we come here to get supplies, even the centre's store are empty. Medicine has not arrived yet.... then you can't wait ...we don't have any other alternative [frustra- tion]' (FGD3, Q)

The shortage of drugs and equipment was said to result in the CHWs relying more on household health care practices and prevention tools.

'We must rely on "domestic heath care" we don't have any
medicine. For example, if a child has fever we put the child
in a sheltered place and rub his body with a wet cloth.
That's how we control fever' (FGD3, S)

According to CHWs, despite good performance on their behalf, major reasons for decreased numbers of patients visiting them, were that mothers were met with a lack of medicines. The community's and the mothers' expectations of delivering treatment to children was perceived as stressful to the CHWs: 


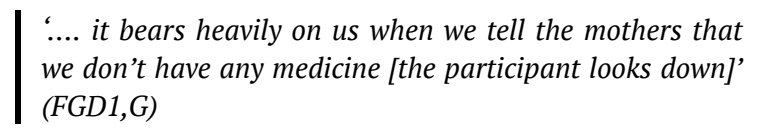

While some CHWs expressed receiving good supervision, others spoke of insufficient supervisions in relation to their workload and responsibilities. Supervision was not always described as a motivator.

'The main problem is the lack of encouragement and motivation from our superiors' (FGD2, M)

\section{INADEQUATE KNOWLEDGE AND AWARENESS}

According to the CHWs, refresher training sessions are not carried out regularly. They expressed that they only address certain childhood illnesses for children under five and have inadequate knowledge on how to treat other child health conditions.

\section{'....our patients are children under five and our training focuses on them...children also come to us with wounds and injuries..."we are not trained for this"' (FGD1,E)}

The participants said some mothers, preferred HC due to mistrust towards the CHWs ability to deliver quality health care. Although the community in general trusted medical treatment, the CHWs, in particular, discussed how mothers had a strong faith in medicines dispensed as syrup vs tablets.

\section{'Syrup especially is trusted by many mothers as a med- icine... we try to convince the mothers that both are the same [laughter]' (FGD1,C)}

Mother's health care seeking behaviors were mentioned as barriers often resulting in delay in seeking appropriate health care.

'Challenges of the program regarding the mothers are not seeking treatment in time...' (FGD1,B)

The CHWs also spoke of children being neglected or not given medical treatment

'They just keep the sick child at home. There are some
mothers who lack awareness...they don't follow our ad-
vice' (FGD1,C)

\section{JUGGLING MULTIPLE ROLES WITH NO PAY}

The CHWs were housewives or farmers, and were mothers with many household duties and responsibilities. The challenge of being a mother, a wife and a CHW were said to be hard to balance, especially when they did not have the support of a husband.

'I'm a mother and on top of that I have many domestic chores to fulfill. This is a big obstacle in my work' (FGD2,B)

Although CHWs in all FGDs took pride in their work, there were many concerns regarding being a voluntary health worker with no financial compensations. They had added responsibilities and had to spend their own money for transportation to the HC to collect drugs. All these fac- tors were said to have influenced their motivation. Yet, the CHWs conveyed it to be important to continue to work for the sake of the community.

'A small allowance gives hope just like the first rain gives the farmer hope...we have taken the additional responsibility of being a CHW ... and all this without compensation' (FGD2,P)

\section{DISCUSSION}

This study explored Eritrean CHWs perceptions and experiences of benefits and challenges of community- based CIMCI/iCCM program in the Maekel region through FGDs. This study also reveals a deeper understanding of CHWs perceptions of implementation experiences and the health care system as well as suggestions as to what is needed for more effective community health outcomes in the Eritrean context. Main findings identified that despite several benefits of the program, these benefits seemed to be hampered by constrains in delivering services, resources, and supervision. Not much is known about the community-based child health program in Eritrea, however, the benefits mentioned by the CHWs seem to match the program goals and are reflected in other studies from Sub-Saharan Africa (SSA) in Uganda, Ghana, Zambia and Malawi. ${ }^{19-25}$

Community support and trust emerged as important motivators for the CHWs in this study and was expressed in all three FGDs. Similar findings were found in other studies including countries such as Ghana, Sierra Leone, Tanzania and Uganda. ${ }^{24-27}$ Although CHWs are suggested to have the potential to reduce child mortality, they often lack needed resources. ${ }^{26}$ Noteworthy, is that all CHWs in this study agreed that resources such as medical supplies, equipment and drugs were heavily insufficient. Similar challenges were also found in other settings such as Tanzania and Ethiopia. ${ }^{26,28}$ In the neighboring country Ethiopia, the CHWs are called Health extension workers (HEWs), and in a study exploring their experiences revealed that the HEWs also experience resource gaps and limited supportive supervisors. ${ }^{28}$

In adopting the program, C-IMCI/iCCM, Eritrea has developed necessary element to deliver community health outcomes. Yet, this rather young country is still in the process of building up and stabilizing its health care system. ${ }^{5,6}$ The WHO /UNICEF are associated with communitybased program where community members do the work by providing care in their own settings. ${ }^{27}$ This suggest that community participation shapes program implementation and facilitation. ${ }^{27}$ This study strongly indicates that the way the international program is designed, with a top-down approach, is not working to its full potential. The study also points to the fact- that the program will be more applicable if the challenges the CHWs face are considered. During data analysis, also shown in our adopted framework, it was clear that the program did not account for factors the CHWs had in their struggle to balance different roles. At home, the CHWs had to negotiate and justify their choice of volunteer work.

Drug availability, clinical supervision and support is necessary for the success of the program. ${ }^{24}$ However, the in- 
tended goals, to increase access and coverage of appropriate care are being unmet and jeopardized due to program limitations. According to the Eritrean Health sector strategic development plan (HSSD), health worker's distributions has been skewed towards urban areas. ${ }^{29}$ Our suggestion is that the MoH should include CHWs in the HSSD plan in attempting to achieve these goals and engaging the community. For the Eritrean program, by allocating resources, the CHWs as part of a community health teams together with the HC staff can help create systems and building community capacity. ${ }^{30}$ There is need for better communication between the CHWs, the local HC and the focal person at each HC. One-way to handle issues related to the drug supply chain is information sharing using technology to facilitate availability of drugs. If communication and technology can be used it would spare the CHWs the long travel to the HC, they can make the journey when drugs are available. Our suggestions here are also to lower CHWs transportation costs by letting them use public transportation, which is owned by the government, for free when travelling for work-related travels. The MoH can provide documents that proves that they are CHWs.

In this study we also examined the possible effect of mothers strong believes in curative treatment and their perception in quality health care. The CHWs are expected to mobilize and increases program acceptance among community members. ${ }^{31}$ In the FGDs, the CHWs elaborated on the notion of curative treatment being the main factor for establishing trust of medical treatment and the CHWs. However, in this study they expressed feeling poorly prepared to deal with encounters when drugs were regularly out of stock. It seemed they felt personally inadequate when they could not provide drugs. They found themselves often negotiating with the service users to gain back the trust and support. Perceived low quality of care were major reasons why the mothers preferred HC. ${ }^{32}$

Community mistrust and decreasing service utilization will eventually affect the efforts made to raise community awareness. Here, community support and participation are crucial for the legitimacy and effectiveness of the program, the CHWs and their services. One tactic used by the CHWs, was to use household child health care practices that they were taught, and referring the children to the health centers, knowing the shortage of medicines. In other words, more preventive tools and measures were applied rather than curative treatment, according to the guidelines. ${ }^{27}$ In an Ethiopian study, it was also suggested that community members perceived the HEWs to deliver inadequate curative services due to lack of resources. ${ }^{33}$

The CHWs stressed the lack of continuous training. Team-based training, opening hours and regularly meetings with the focal persons, training for better supervision would be worthwhile suggestions. Workshops with focus on problem -solving, support groups and distance -learning sessions can also be conducted in a matter that includes CHWs, their families and community members. Currently there are two CHWs at each village. By training more CHWs, they could share the workload and the training can involving the CHWs as mentors.

In the Eritrean context, a discussion is needed, focusing on coordination, communication and innovation. ${ }^{34}$ A pro- gram is only as strong as its weakest link. Considering the crucial importance of the CHW as the frontline worker of the healthcare system, their lack of value threatens the delivery of health care interventions for the population it was built for. The findings in this study suggest that the Maekel region is struggling with delivering the program within an existing fragmented health care system with insufficient resources. ${ }^{5,6}$ The strong dedication to give back and serve the community as a volunteer is laudable, however this does not necessarily contradict the CHWs desire to receive monetary support. The CHWs are left to negotiate between the desires and satisfaction of caring for and serving the collective and the difficult conditions of being unpaid. Nevertheless, this will have implications on the implementation of the program. ${ }^{35}$ This is an important factor to consider regarding retention of these essential CHWs.

Conducting this study, involving FGDs has given the CHWs voice to be heard. This study has underscored the important role of CHWs and revealed some of their satisfaction with the role but more importantly it has identified a number of issues that limit their ability to provide essential frontline care. Further research is needed to explore how to overcome these problems of accessing the community, developing the supply chain and assessing the feasibility of the program in the region.

Using the adopted framework illuminated CHWs perspectives and showed were the benefits and the challenges were brought up. However, this research found that the framework fell short of describing what it really meant for the CHWs to be involved in such a program. It was clear that navigating the program and the health care system and negotiating for support and financial compensation were not mutually exclusive but were rather enmeshed.

\section{STRENGTHS AND LIMITATIONS}

The primary investigator is originally from Eritrea, a Tigrinya speaker and a therefore culturally attuned, providing a valuable position as the insider-outsider perspective. ${ }^{36}$ Being an insider-outsider has a multiplicity of influences of which it is difficult to determine precisely however the overall strength of cultural competence and language abilities enables a depth not available to outsider. ${ }^{36}$ The intention of the researcher was to hear all participants, as the topic has not been studied from CHWs perspectives in the region, prior to this study. Having only one male CHWs participating in the study, made it possible to explore his experiences. Yet, it is hard to know how he affected the FGDs.

One limitation of the study may be that the CHWs were only accessed through the focal persons. However, since the CHWs live in rural villages and are difficult to reach, it would have been extremely difficult for the research team to locate them. Additional limitation may be that the participants may have exaggerated when presenting the challenges or ignored to mention additional benefits in the hopes of receiving program incentives. Overall, peer-briefing, prolonged engagement in the Maekel region, participant observation in the field, and member checking heightened trustworthiness of the data. ${ }^{36}$ 


\section{CONCLUSION}

Overall, this study highlights the perceptions and experiences of CHWs in the Maekel region revealing both positive and challenging aspects of the community programs. The findings conclude that despite the positive aspects mentioned that align with the program goals, there were sufficient challenges that undermined the programs provision of care, such as constraints in delivering services, limited resources and ineffective supervision. In order to cope, the CHWs were forced to navigate the system barriers and negotiate for support and resources as unpaid volunteers. This study also provides a deeper understanding of the factors associated with CHWs motivation. For improved effectiveness and sustainability of programs, future studies would do well to focus on how to tackle theses identified challenges within the current health care system.

\section{ACKNOWLEDGEMENTS}

We would like to thank the Ministry of Health in Eritrea. We are also grateful to the CHWs who willingly accepted to be interviewed. Ethical clearance was obtained from the Ministry of Health's Ethical committee in Asmara, Eritrea.

\section{FUNDING}

The fieldwork for this study was funded by The Swedish In- ternational Development Cooperation Agency by a Minor field studies scholarship

\section{AUTHORSHIP CONTRIBUTIONS}

Designed the study: $\mathrm{JTM}^{1}$ and $\mathrm{JT}^{2}$. Collected the data: JTM ${ }^{1}$. Analyzed the data: JTM $^{1}$ with feedback from $\mathrm{JT}^{2}$. Wrote the first draft of the manuscript: JTM ${ }^{1}$. Contributed to the writing of the manuscript: JTM $^{1}$ and $\mathrm{JT}^{2}$

\section{CONFLICT OF INTEREST}

The authors completed the Unified Competing Interest form at www.icmje.org/coi_disclosure.pdf (available upon request from the corresponding author) and declare no conflicts of interest.

\section{CORRESPONDENCE TO:}

Jordanos Tewelde McDonald, MSc, MPH Doctoral Student, Karolinska Institutet

Department of Global Public Health, Global and Sexual Health Research Group

Tomtebodavägen 18A, 17177 Stockholm

Jordanos.tewelde@ki.se

Submitted: April 06, 2021 GMT, Accepted: May 02, 2021 GMT 


\section{REFERENCES}

1. UN Inter-Agency Group for Child Mortality

Estimation: Levels and Trends in Child Mortality Report. Accessed December 27, 2020. https://childmortality.or g/resources

2. World Health Organization and United Nations' Children's Fund. Joint statement: Integrated community case management- an equity-focused strategy to improve access to essential treatment services for children. Accessed December 28, 2020. htt ps://www.who.int/maternal_child_adolescent/docume nts/iccm_service_access/en/

3. World Health Organization. The Country Cooperation Strategy: Eritrea 2018. Accessed November 13, 2020. https://apps.who.int/iris/bitstrea $\mathrm{m} /$ handle/10665/137169/ccsbrief eri en.pdf?sequenc e=1\&isAllowed $=\mathrm{y}$

4. Kebedom A, Teclebirhan T, Beyene W. Use Integrated Management of Childhood Illnesses guidelines: a need for adaption for use in Gindae Hospital, Eritrea. J Erit Med Assoc. 2009;4(1). doi:10.4 314/jema.v4i1.52119

5. The State of Eritrea, The Ministry of Health. National child health strategy 2014-2018. Published online 2013.

6. Overseas Development Institute. Development progress. Progress in health in Eritrea: cost-effective inter-sectoral interventions and a long-term perspective. Accessed November 30, 2020. https://od i.org/en/publications/progress-in-health-in-eritrea-c ost-effective-inter-sectoral-interventions-and-a-lon g-term-perspective/

7. The State of Eritrea: Ministry of Health. National health policy. Accessed July 20, 2020. http://www.wh o.int/medical devices/survey_resources/health techn ology_national_policy_eritrea.pdf

8. World Health Organization. Handbook: IMCI Integrated Management of Childhood Illness. Accessed May 6, 2020. https://apps.who.int/iris/handle/10665/4 $\underline{2939}$

9. Chopra M, Binkin JN, Mason E. Integrated management of childhood illnesses: what have we learned and how can it improve. Arch Dis child. 2012;97(4):350-354.

10. Thompson ME, Harutyunyan TL. Impact of a community-based integrated management of childhood illnesses (IMCI) programme in Gegharkunik, Armenia. Health Policy and Planning. 2009;24(2):101-107.
11. Nguyen Kim DT, Leung KK, McIntyre L. Does Integrated management of childhood illnesses (IMCI) training improve the skills of health workers? A systematic review and meta-analysis. PLOS ONE. 2013;8(6):e66030.

12. Miller PN, Amouzou A, Tafesse M. Integrated Community Case Management of Childhood Illness in Ethiopia: Implementation strength and Quality of Care. Am J Med Hyg. 2014;91(2):424-434.

13. Diaz T, Aboubaker S, Young M. Current scientific evidence for integrated community case management in Africa: Findings from the iCCM evidence symposium. J Glob Health. 2014;4(2).

14. Olaniran A, Smith H. Who is a community health worker? -a systematic review of definitions. Global Health Action. 2017;10:1.

15. Green J, Thorogood N. Qualitative Methods for Health Research. SAGE Publication Ltd; 2009.

16. Duggleby M. What about focus group interaction data? Qualitative Health Res. 2005;15(6):832-840.

17. United Nations' Children's Fund. Community Health Worker + Framework. Accessed February 13, 2016. http://www.unicef.org/innovation/innovation 8 1728.html

18. Graneheim UH, Lundman B. Qualitative content analysis in nursing research: concept, procedure and measurement to achieve trustworthiness. Nurse Educ Today. 2004;24(2):105-112.

19. Mubiru D, Byabasheija R, Bwanika JB, et al. Evaluation of Integrated community case management in eight Districts of Central Uganda. Baradaran HR, ed. PLoS ONE. 2015;10(8):137. doi:10.1 371/journal.pone.0134767

20. Miller NP, Amouzou A, Tafesse M, et al. Integrated Community Case Management of Childhood Illness in Ethiopia: Implementation strength and Quality of Care. Am J Med Hyg. 2014;91(2):424-434. doi:10.4269/ ajtmh.13-0751

21. Shaw B, Amouzou A, Miller NP, et al. Determinants of Utilization of Health Extension Workers in the Context of Scale-Up of Integrated Community Case Management of Childhood Illnesses in Ethiopia. Am J Trop Med Hyg. 2015;93(3):636-647. oi:10.4269/ajtmh.14-0660 
22. Seidenberg PD, Hamer DH, Iyer H, et al. Impact of Integrated Community Case Management on HealthSeeking Behavior in Rural Zambia. Am J Med Hyg. 2012;87(5):105-110. doi:10.4269/ajtmh.2012.11-0799

23. Callaghan-Koru JA, Hyder AA, George A, et al. Health Workers' and Managers' Perceptions of the Integrated Community Case Management Program for Childhood Illness in Malawi: The Importance of Expanding Access to Child Health Services. Am J Med Hyg. 2012;87(5):61-68. doi:10.4269/ajtmh.2012.11-06 $\underline{65}$

24. Daniels K, Sanders D, Daviaud E, Doherty T. Valuing and Sustaining (or Not) the Ability of Volunteer Community Health Workers to Deliver Integrated Community Case Management in Northern Ghana: a qualitative study. Molyneux S, ed. PLoS ONE. 2015;10(6):1371. doi:10.1371/journal.pon e. 0126322

25. Buchner DL, Brenner JL, Kabakyenga J, et al. Stakeholders' Perceptions of Integrated Community Case Management by Community Health Workers: A post-intervention Qualitative Study. Sambhara S, ed. PLoS ONE. 2014;9(6):e98610. doi:10.1371/journal.pon $\underline{\mathrm{e} .0098610}$

26. Greenspan JA, McMahon SA, Chebet JJ, Mpunga M, Urassa DP, Winch PJ. Sources of community health worker motivation: a qualitative study in Morogoro Region, Tanzania. Hum Resour Health. 2013;11(1):52. doi:10.1186/1478-4491-11-52

27. Rifkin BS. Translating Rhetoric to Reality: A review of community participation in health policy over the last 60 years. Accessed April 27, 2018. http s://www.wzb.eu/sites/default/files/u35/rifkin_2012_rh etoric to reality_a_review_of_cp_and health_policy.pd f

28. Assefa Y, Gelaw YA, Hill PS, Taye BW, Van Damme W. Community health extension program of Ethiopia, 2003-2018: successes and challenges toward universal coverage for primary healthcare services. Global Health. 2019;15(1):24. doi:10.1186/s12992-01 9-0470-1
29. The State of Eritrea, The Ministry of Health. Health sector strategic development plan 2010-2014.

30. Balcazar H, Rosenthal EL. Community health workers can be a public health force for change in the United States: Three actions for a new paradigm.

31. Olaniran A, Smith H, Unkels R, Bar-Zeev S, van den Broek N. Who is a community health worker? - a systematic review of definitions. Global Health Action. 2017;10(1):1272223. doi:10.1080/16549716.2017.1272 $\underline{223}$

32. Chol C, Hunter C, Debru B, Haile B, Negin J, Cumming RG. Stakeholders' perspectives on facilitators of and barriers to the utilisation of and access to maternal health services in Eritrea: a qualitative study. BMC Pregnancy Childbirth. 2018;18(1):35. doi:10.1186/s12884-018-1665-9

33. Tilahun H, Fekadu B, Abdisa H. Ethiopia's health extension workers use of work time on duty: time and motion study. Health Policy and Planning.

2017;32(3):320-328. https://academic.oup.com/heapo l/article/32/3/320/2555444

34. Valaitis RK, Carter N, Lam A, Nicholl J, Feather J, Cleghorn L. Implementation and maintenance of patient navigation programs linking primary care with community-based health and social services: a scoping literature review. BMC Health Serv Res. 2017;17(1):116. doi:10.1186/s12913-017-2046-1

35. Yansaneh AI, George AS, Sharkey A. Determinants of Utilizations of Health Extension Workers in the Context of Scale-Up of Integrated Community Case Management of Childhood Illnesses in Ethiopia. J Community Health. 2015;10:1007.

36. Ergun A, Erdemir A. Negotiating Insider and Outsider Identities in the field: "Insider" in a Foreign Land; "outsider" in One's Own Land. Field Methods. 2010;22(1):16-28. doi:10.1177/1525822x09349919 